\title{
Etude expérimentale du lavage de l'iode par la pluie*
}

\author{
C. CAPUT, H. CAMUS, D. GAUTHIER, Y. BELOT**
}

(Manuscrit reçu le 11 juin 1992)

\begin{abstract}
RÉSUMÉ Des expérimentations de terrain ont été effectuées pour déterminer le facteur de lavage par la pluie de l'iode élémentaire à l'état de vapeur. Elles ont consisté à émettre une quantité connue d'iode stable et à recueillir le dépôt humide dans des collecteurs convenablement disposés pour évaluer la fraction du panache entraînée par la pluie. Il a été montré que des précautions particulières devaient être prises pour éviter une superposition du dépôt direct de la vapeur d'iode par réaction sur les surfaces collectrices (dépôt sec) et du dépôt indirect de l'iode entraîné par la pluie (dépôt humide). Les résultats obtenus en ce qui concerne le facteur de lavage $\left(8,2 \times 10^{-5} \mathrm{~s}^{-1}\right.$ en moyenne) valident les valeurs théoriques habituellement utilisées et montrent que tout se passe dans les conditions de ces expériences comme si la captation de l'iode par les gouttes de pluie était irréversible.
\end{abstract}

ABSTRACT Field experiments were performed to determine the washout coefficient of iodine vapour scavenged by rain. They consisted in emitting a known amount of stable iodine and sampling the wet deposit in collectors conveniently placed to evaluate the fraction of plume scavenged by rain. It was shown that precautions are to be taken to avoid a superposition of direct deposition of iodine vapour by reaction on collector surfaces (dry deposition) and indirect deposition by rain scavenging (wet deposition). The data obtained (mean value $=8.2 \times 10^{-5} \mathrm{~s}^{-1}$ validate the theorical values currently used and indicate that, under the conditions of these experiments, iodine uptake by rain droplets is apparently irreversible.

\section{Introduction}

Le lavage de l'iode par la pluie est habituellement caractérisé par la fraction d'iode entraînée par unité de temps appelée facteur ou coefficient de lavage $(\Lambda)$. Une étude bibliographique nous a montré que les facteurs de lavage de l'iode moléculaire actuellement utilisés dans les modèles proviennent exclusivement de considérations théoriques et sont basés, en particulier, sur l'hypothèse d'une absorption irréversible de l'iode par les gouttes de pluie [2]. Quelques auteurs ont avancé des arguments théoriques plaidant en faveur d'une dissolution réversible de l'iode et ont pensé que les facteurs de lavage usuels pouvaient être, de ce fait, largement surestimés [1,4]. Pour étayer ou infirmer cette supposition, il fallait se rapporter à l'expérience. Malheureusement, les données dispo-

* Etudes effectuée dans le cadre d'un protocole passé entre CEA / IPSN et EDF / DE.

* Commissariat à l'énergie atomique, Institut de protection et de sûreté nucléaire, Département de protection de l'environnement et des installations (DPEI), BP 6, 92265 Fontenay-aux-Roses Cedex, France. 
nibles étaient quasi inexistantes, elles se réduisaient, en fait, à trois valeurs de facteur de lavage [3] comprises entre $1,5 \times 10^{-7}$ et $2,5 \times 10^{-6}$ $\mathrm{s}^{-1}$, obtenues seulement dans des conditions de pluviosité faible (intensités de 0,4 à $0,5 \mathrm{~mm} \mathrm{~h}^{-1}$ ). Ces valeurs étaient, certes, beaucoup plus basses que les valeurs habituellement admises, mais en nombre trop restreint pour autoriser des conclusions valables. De nouvelles expériences de terrain ont donc été effectuées pour valider ou invalider les quelques observations disponibles, choisir en faveur d'une absorption réversible ou irréversible, et éventuellement réviser dans un sens plus réaliste les valeurs couramment utilisées sur la base de considérations purement théoriques.

\section{Choix des sites}

Dans une première phase, le site de Brennilis (Monts d'Arrée) a été choisi en raison de la pluviosité importante qui y règne statistiquement, et de certaines caractéristiques orographiques apparemment favorables : présence d'un point haut pour implanter le dispositif d'émission, dominant d'une hauteur de $30 \mathrm{~m}$ deux routes permettant l'installation des collecteurs sous les vents dominants, à des distances moyennes du générateur de 320 et $1720 \mathrm{~m}$. En raison d'un écoulement des filets d'air perturbé par une dénivellation brutale du sol sous le vent du générateur, il s'est avéré, comme on le verra plus loin, que des rabattements du panache sur les collecteurs provoquaient un dépôt sec parasite venant se superposer au dépôt humide objet de l'étude. Un autre emplacement voisin a donc été choisi ultérieurement, le site de la station de Télédiffusion de France de Roc'h Trédudon, au sommet d'une colline en pente douce engendrant un écoulement de l'air moins turbulent. Le dispositif d'émission était alors disposé sur un mât télescopique de $12 \mathrm{~m}$, et les collecteurs de précipitations répartis sur des arcs de cercle de rayon inférieur à $50 \mathrm{~m}$, de telle sorte qu'ils ne soient pas touchés par le panache d'iode.

\section{Méthodes expérimentales}

Chaque expérience était effectuée en présence de précipitations caractérisées par leur intensité, le spectre dimensionnel des gouttes, le $\mathrm{pH}$, et la température de l'eau de pluie. Elle consistait à émettre dans l'atmosphère une quantité connue d'iode stable sous forme de vapeur d'iode moléculaire (de 4,5 à $100 \mathrm{~g}$ ). La durée de l'émission était généralement de 2 min, sauf lors de 6 essais effectués à Roc'h Trédudon, où elle a été portée à $1 \mathrm{~h}$, en vue de mettre en évidence un éventuel effet de la concentration d'iode dans l'air sur le facteur de lavage. Cette vapeur d'iode moléculaire était obtenue par chauffage de paillettes d'iode contenues dans une ampoule de pyrex soumise à un flux d'air à $400^{\circ} \mathrm{C}$ (émissions de $2 \mathrm{~min}$ ), ou $200^{\circ} \mathrm{C}$ (émissions de $1 \mathrm{~h}$ ). L'ensemble du générateur 
était abrité de la pluie, tout en étant balayé par le vent. L'eau de pluie était échantillonnée sous le vent de l'émission à l'aide de collecteurs de précipitation régulièrement espacés et disposés soit le long des chemins les plus appropriés (Brennilis), soit en arcs de cercle et à des distances de l'émission de 20 à $50 \mathrm{~m}$ (Roc'h Trédudon), de manière à englober l'intégralité de la largeur du panache. A l'issue de chaque essai, on mesurait le volume de l'eau collectée en chaque point ainsi que sa concentration en iode. Celle-ci était mesurée par une méthode de chromatographie ionique (Dionex), basée sur une préconcentration de l'échantillon et une détection électrochimique très sensible de l'iode sous forme d'iodure. Des essais préliminaires ont montré que l'iode contenu dans les eaux de pluie recueillies au cours de nos expériences était entièrement à l'état d'iodure. Les bruits de fond d'iode mesurés hors de l'emprise du panache, compris entre 0,5 et $1 \mu \mathrm{g}^{-1}$, étaient soustraits des valeurs brutes obtenues dans les échantillons collectés sous le panache.

Le lavage de l'iode par la pluie est caractérisé par un coefficient de lavage (ou facteur de lavage), habituellement noté $\Lambda$ (en $\mathrm{s}^{-1}$ ), qui est la fraction d'jode entraînée par unité de temps. Pour obtenir expérimentalement le facteur de lavage, on calcule pendant combien de temps le panache passe au-dessus des collecteurs, et finalement la fraction de panache entraînée par unité de temps. Si l'on appelle $W$ le dépôt moyen par unité de surface le long de l'arc échantillonné $\left(\mathrm{g} \mathrm{m}^{-2}\right), L$ la longueur de cet arc $(\mathrm{m})$ englobant l'ensemble de la largeur du panache, $u$ la vitesse moyenne du vent $\left(\mathrm{m} \mathrm{s}^{-1}\right)$ et $Q$ la quantité totale d'iode lâchée pendant l'expérience $(\mathrm{g})$, le facteur de lavage exprimé en $\mathrm{s}^{-1}$ est :

$$
\Lambda=W L U / Q
$$

\section{Résultats et discussion}

\subsection{Expérience de Brennilis}

Les valeurs des facteurs de lavage obtenues à Brennilis (fig. 1) ne sont pas corrélées à l'intensité de précipitation. Elles sont comprises entre $2,1 \times 10^{-5}$ et $4,0 \times 10^{-4} \mathrm{~s}^{-1}$ avec une moyenne de $2 \times 10^{-4} \mathrm{~s}^{-1}$ et sont, dans l'ensemble, nettement plus élevées que les valeurs théoriques habituellement utilisées, en particulier pour les faibles intensités de précipitation. De ce fait, nous avons été amenés à suspecter que l'iode recueilli ne provenait pas uniquement d'un entraînement de l'iode par la pluie, mais résultait aussi d'un dépôt de l'iode moléculaire gazeux sur les parois des collecteurs de précipitation. L'existence de ce dépôt sec parasite fut confirmée dans des expériences analytiques ultérieures réalisées en exposant des collecteurs de divers types dans des panaches d'iode en l'absence et en présence de précipitation. Nous avons observé que l'iode moléculaire gazeux, très réactif, se déposait par voie sèche sur les collecteurs de précipitation lorsque ceux-ci se trouvaient dans le panache. La présence d'un film d'eau sur la paroi interne de l'entonnoir 
et du récipient qui collecte la pluie accentuait ce phénomène en augmentant le dépôt sec d'un facteur 2 à 4 . La nature du matériau constituant les collecteurs importait relativement peu dès lors que les surfaces étaient mouillées, comme l'ont montré des essais réalisés avec les différents types d'entonnoirs susceptibles d'être utilisés (acier inoxydable, verre, polyéthylène, polypropylène, téflon).

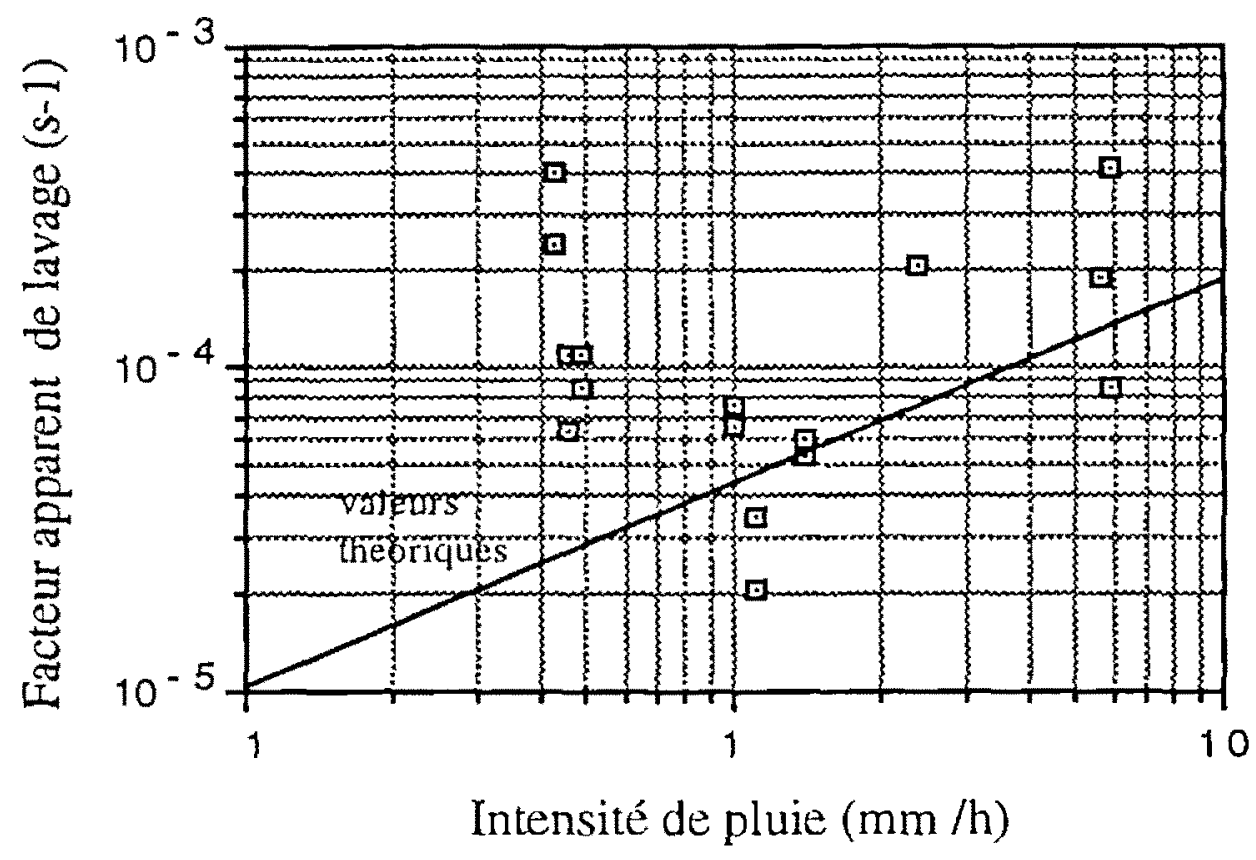

Fig. 1. - Facteurs apparents de lavage de l'iode obtenus à l'issue de 16 expériences effectuées à Brennilis. La ligne en trait plein représente les valeurs théoriques obtenues en supposant que la captation de l'iode par les gouttes de pluie est irréversible. Le pH de reau de pluie est compris entre 5,5 et 6,0 .

Apparent iodine washout coefficients obtained from 16 field experiments at Brennilis. The full line represents the theoretical values obtained assuming irreversible iodine uptake by rain drops. Rain water pH range: $5.5-6.0$.

\subsection{Expériences de Roc'h Trédudon}

La seule solution envisageable pour déterminer la véritable valeur du facteur de lavage était de placer les collecteurs de précipitation à faible distance d'un point d'émission surélevé dans la zone où le panache ne touche pas encore le sol, les collecteurs recevant alors la pluie qui a traversé le panache, sans être exposés à un dépôt direct par voie sèche. Une expérience à blanc a d'abord été réalisée en l'absence de précipitation, mais par fort vent d'ouest accompagné d'un niveau élevé de turbulence (conditions anémométriques accompagnant la plupart des pluies dans cette région), et par forte humidité permettant de maintenir mouillés les entonnoirs des collecteurs. II a été observé que jusqu'à une distance de la source au moins égale à $50 \mathrm{~m}$, aucun dépôt sec n'était recueilli. Par surcroît de précaution, au cours de chacun des essais ultérieurs, un entonnoir préalablement mouillé par l'eau de pluie a été installé à l'abri 
de la pluie sur l'arc de prélèvement et à proximité de l'axe du panache, pour mettre en évidence un éventuel dépôt sec, ce qui n'a jamais été constaté lors des différentes expériences réalisées; 17 déterminations de facteurs de lavage ont été effectuées pour des intensités de précipitation allant de 0,8 à $6,3 \mathrm{~mm} \mathrm{~h}^{-1}$ et des vitesses de vent comprises entre 2,5 et $18,3 \mathrm{~m} \mathrm{~s}^{-1}$ englobant les conditions les plus fréquentes sous nos latitudes. Les facteurs de lavage (fig. 2) sont compris entre $1,5 \times 10^{-5}$ et $3 \times 10^{-4} \mathrm{~s}^{-1}$ avec une moyenne de $8,2 \times 10^{-5} \mathrm{~s}^{-1}$. Cette valeur moyenne, non affectée par un dépôt sec parasite, est deux fois plus faible que celle tirée des expériences de Brennilis.

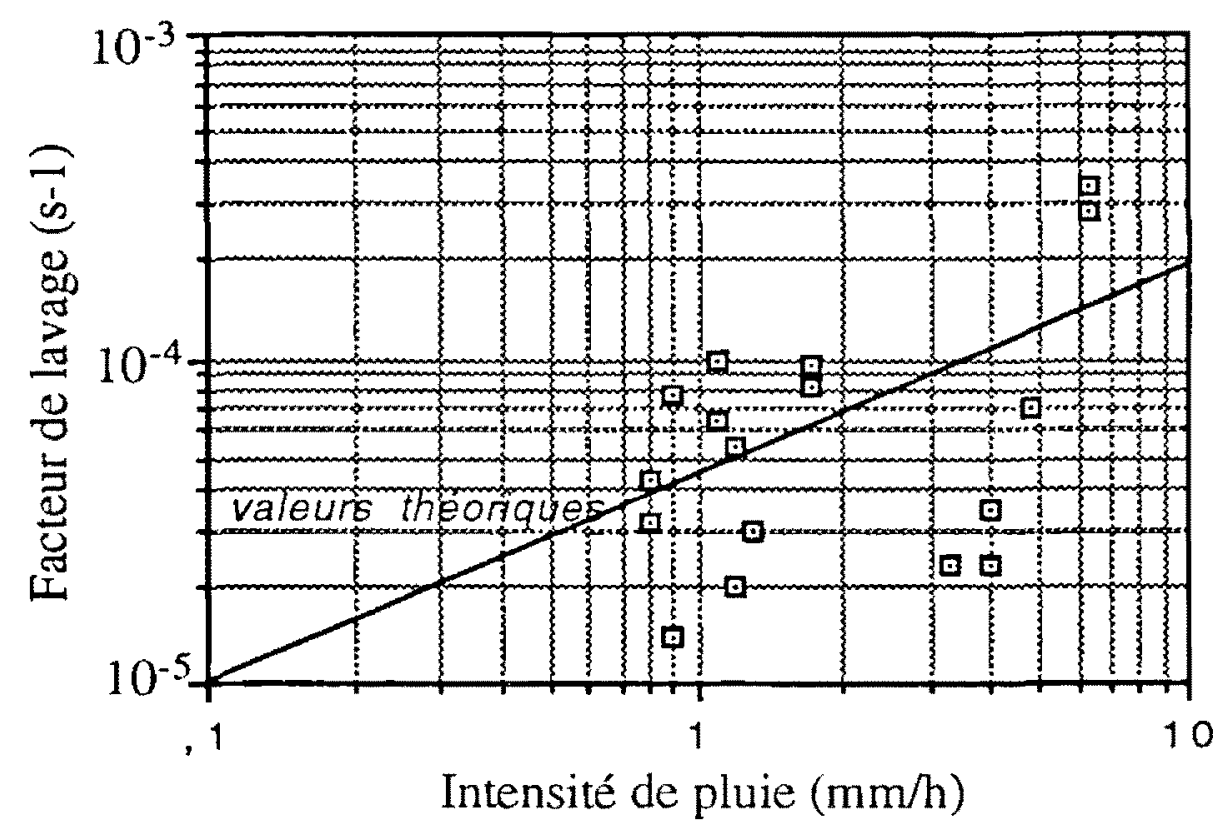

Fig. 2. - Facteurs apparents de lavage de l'iode obtenus à l'issue de 17 expériences effectuées à Roc'h Trédudon. La ligne en trait plein représente les valeurs théoriques obtenues en supposant que la captation de l'iode par les gouttes de pluie est irréversible. Le pH de l'eau de pluie est compris entre 5,5 et 6,0.

Apparent iodine washout coefficients obtained from 17 field experiments at Roc'h Tredudon. The full line represents the theoretical values obtained assuming irreversible iodine uptake by rain drops. Rain water pH range: $5.5-6.0$.

\subsection{Interprétation des résultats}

Au cours de nos expériences, la quantité d'iode stable émise dans l'atmosphère était suffisamment faible pour que la concentration de l'iode dans la pluie ne soit pas modifiée dans des proportions considérables. Cependant, on a mesuré dans l'eau de pluie prélevée en dessous de nos panaches expérimentaux des concentrations d'iode comprises entre quelques $\mu \mathrm{g} \mathrm{I}^{-1}$ et quelques dizaines de $\mu \mathrm{g} \mathrm{I}^{-1}$, que l'on peut comparer au bruit de fond naturel d'environ $1 \mu \mathrm{g} \mathrm{I}^{-1}$. Si l'on distingue les résultats des 6 expériences où la concentration d'iode dans l'eau reste très proche du bruit de fond, et ceux des expériences où la concentration est locale- 
ment multipliée par un facteur 10 ou plus, on n'observe aucune différence significative entre les facteurs de lavage correspondants ; la concentration massique de l'iode moléculaire ne semble donc pas avoir une influence importante sur le lavage, tout au moins dans le domaine des concentrations rencontrées au cours de nos expériences.

Les résultats obtenus sont indépendants de la température (6 à $20^{\circ} \mathrm{C}$ ) et du $\mathrm{pH}$ de l'eau de pluie $(5,5$ à 6,0$)$ mesurés lors de nos essais. Ils sont assez dispersés et ne sont pas corrélés de manière très nette avec l'intensité de précipitation, elle-même étroitement liée à la taille des gouttes. La dispersion observée qui masque l'effet de l'intensité de précipitation et des autres paramètres caractérisant la pluie provient, sans doute, de l'écart qui existe entre les conditions atmosphériques idéalisées qui nous ont servi à déterminer les facteurs de lavage moyens et les conditions atmosphériques réelles. La formule employée suppose que la pluie tombe verticalement et que le champ de vitesse de vent est uniforme dans le temps et dans l'espace, hypothèses très simplificatrices. L'existence de turbulences rend, sans doute, la détermination du facteur de lavage imprécise ; or, l'intensité et le spectre dimensionnel des turbulences sont généralement très importants en présence de précipitations autres que les petites pluies de front chaud. II n'en reste pas moins que les nombreuses valeurs obtenues sont en moyenne assez proches des valeurs que l'on peut obtenir théoriquement en supposant que la captation de l'iode moléculaire par les gouttes d'eau se fait de manière irréversible. Cette irréversibilité implique que la transformation de l'iode moléculaire volatil en iodures non volatils s'effectue plus rapidement qu'il n'est théoriquement prévu [3] pour des solutions de $\mathrm{pH}$ inférieur à 6 . La cinétique de transformation de l'iode moléculaire dissous en iodures non volatils pourrait être accélérée, sous réserve d'inventaire, par la présence de composés réducteurs tels que le dioxyde de soufre par exemple.

\subsection{Importance relative du dépôt humide et du dépôt sec}

Les deux séries d'expériences réalisées à Brennilis et à Roc'h Trédudon ont montré que lorsque le panache touche le sol, le dépôt observé pendant les épisodes de pluie est dû à la superposition d'un dépôt sec de la vapeur d'iode élémentaire, et d'un dépôt humide résultant de l'entraînement de l'iode par la pluie. Le dépôt sec peut quelquefois être plus important que le dépôt humide, notamment pour les faibles intensités de précipitation. On peut se demander comment varie le rapport du dépôt sec au dépôt humide dans les différentes conditions d'un dépôt réel en situation accidentelle.

Pour comparer les importances relatives des dépôts sec et humide, on peut utiliser comme critère les vitesses de dépôt qui caractérisent ces deux mécanismes. Ces deux vitesses de dépôt sont définies de manière identique comme le rapport de la quantité déposée par unité de surface à la concentration intégrée au voisinage du sol. En admettant que la distribution des concentrations d'iode dans l'air peut être représentée par la 
distribution gaussienne classique, on peut montrer facilement que la vitesse de dépôt humide $V_{w}\left(\right.$ en $\mathrm{m} \mathrm{s}^{-1}$ ) est liée au facteur de lavage $\Lambda$ (en $\left.s^{-1}\right)$ par la relation suivante :

$$
V_{w}=1,25 \wedge \sigma_{z} \exp \left(H^{2} / 2 \sigma_{z}^{2}\right)
$$

où $H$ et $\sigma_{z}$ (en $\mathrm{m}$ ) sont respectivement la hauteur du point d'émission audessus du sol et l'écart-type de la distribution verticale des concentrations, qui augmente avec la distance à la source. II apparaît d'après la formule précédente que la vitesse de dépôt humide est très grande à proximité d'une source élevée, à cause du terme exponentiel figurant dans l'expression (sous réserve, bien entendu, que la pluie qui tombe obliquement ait traversé le panache, ce qui implique de se trouver à une certaine distance de la source élevée, dépendant de la vitesse du vent et de la hauteur d'émission). Cette vitesse de dépôt humide diminue en fonction de la distance à la source pour atteindre un minimum et augmenter ensuite.

Dans le cas, par exemple, d'un facteur de lavage égal à $5 \times 10^{-5} \mathrm{~s}^{-1}$, d'une source située à une hauteur de $30 \mathrm{~m}$, la vitesse de dépôt humide à $1000 \mathrm{~m}$ de la source est égale à $3 \times 10^{-3} \mathrm{~m} \mathrm{~s}^{-1}$. Si on la compare à une vitesse de dépôt sec de $1 \times 10^{-2} \mathrm{~m} \mathrm{~s}^{-1}$, plausible pour un gaz très réactif tel que l'iode moléculaire, on voit que le dépôt sec est approximativement 3 fois plus important que le dépôt humide. En revanche, à proximité immédiate de la source, ou bien à des distances au contraire très grandes, la vitesse de dépôt humide peut atteindre des valeurs très élevées par rapport à la vitesse de dépôt sec. Dépôt humide et dépôt sec sont, bien entendu, à pondérer par la durée relative des épisodes pluvieux.

\section{Conclusions}

Les expériences de lavage de vapeur d'iode par la pluie effectuées sur le terrain ont permis d'aboutir aux conclusions suivantes :

- des précautions doivent être prises pour déterminer le dépôt humide de la vapeur d'iode et éviter toute interférence avec son dépôt sec (dépôt direct sur les parois du collecteur) ;

- les facteurs de lavage obtenus expérimentalement sont en bon accord avec les facteurs théoriques habituellement utilisés ;

- dans les conditions de ces expériences, tout se passe comme si la captation de l'iode élémentaire par les gouttes de pluie était irréversible, ce qui pourrait indiquer une transformation très rapide de l'iode élémentaire en iodures non volatils. Le phénomènes observé pourrait être liée à la composition de l'eau de pluie et en particulier à son contenu en substances réductrices. 


\section{RÉFÉRENCES}

[1] BÖRHINGER I.M., RESELE G., SUTTER H. - Washout of radioiodine : influence of the ambient non-radioactive 1-127 and of droplet-droplet interaction. In : The environmental transfer to man of radionuclides released from nuclear installations, Brussels, 17-21 October 1983. Luxembourg : Communautés européennes, 1984, vol. 1, 117-124.

[2] CHAMBERLAIN A.C. - Aspects of travel and deposition of aerosol and vapour clouds. Report AERE-HP/R-1261, 1955.

[3] ENGELMANN R.J., PERKINS R.W., HAGEN D.I., HALLER W.A. - Washout coefficients for selected gases and particulates. 59th Annual meeting of the Air pollution control association, San Francisco, June 20-24, 1966. BNWL-SA-657, 1966.

[4] HALES J.M. - Fundamentals of the theory of gas scavenging by rain. Atmos. Environ., 1972, 6, 635-659. 